Article

\title{
Competition-Independence Game and Domination Game
}

\author{
Chalermpong Worawannotai ${ }^{1}$ and Watcharintorn Ruksasakchai ${ }^{2, *}$ \\ 1 Department of Mathematics, Faculty of Science, Silpakorn University, Nakhon Pathom 73000, Thailand; \\ worawannotai_c@silpakorn.edu \\ 2 Department of Mathematics, Statistics and Computer Science, Faculty of Liberal Arts and Science, \\ Kasetsart University, Kamphaeng Saen Campus, Nakhon Pathom 73140, Thailand \\ * Correspondence: faaswtr@ku.ac.th
}

Received: 31 January 2020; Accepted: 17 February 2020; Published: 5 March 2020

check for updates

\begin{abstract}
The domination game is played on a graph by two players, Dominator and Staller, who alternately choose a vertex of $G$. Dominator aims to finish the game in as few turns as possible while Staller aims to finish the game in as many turns as possible. The game ends when all vertices are dominated. The game domination number, denoted by $\gamma_{g}(G)$ (respectively $\gamma_{g}^{\prime}(G)$ ), is the total number of turns when both players play optimally and when Dominator (respectively Staller) starts the game. In this paper, we study a version of this game where the set of chosen vertices is always independent. This version turns out to be another game known as the competition-independence game. The competition-independence game is played on a graph by two players, Diminisher and Sweller. They take turns in constructing maximal independent set $M$, where Diminisher tries to minimize $|M|$ and Sweller tries to maximize $|M|$. Note that, actually, it is the domination game in which the set of played vertices is independent. The competition-independence number, denoted by $I_{d}(G)$ (respectively $I_{S}(G)$ ) is the optimal size of the final independent set in the competition-independence game if Diminisher (respectively Sweller) starts the game. In this paper, we check whether some well-known results in the domination game hold for the competition-independence game. We compare the competition-independence numbers to the game domination numbers. Moreover, we provide a family of graphs such that many parameters are equal. Finally, we present a realization result on the competition-independence numbers.
\end{abstract}

Keywords: domination game; competition-independence game

\section{Introduction}

A dominating set of a graph $G$ is a set $S$ of vertices of $G$ such that every vertex in $G$ is an element in $S$ or is adjacent to an element in $S$. The domination number of $G$, denoted by $\gamma(G)$, is the cardinality of a minimum dominating set of $G$. A set $S$ is independent if no two vertices in $S$ are adjacent. The independence number of a graph $G$, denoted by $\alpha(G)$, is the cardinality of a maximum independent set of $G$. An independent dominating set of a graph $G$ is a dominating set of $G$ which is independent. The independent domination number of $G$, denoted by $i(G)$, is the cardinality of a minimum independent dominating set of $G$.

In 2010, Brešar, Klavžar, and Rall [1] first introduced the domination game. The domination game is played on a graph $G$ by two players, Dominator and Staller, who alternately choose a vertex of $G$ in such a way that at least one new vertex is dominated. The game ends when all vertices are dominated. Dominator aims to finish the game in as few turns as possible while Staller aims to finish the game in as many turns as possible. The game domination number, denoted by $\gamma_{g}(G)$ (respectively $\gamma_{g}^{\prime}(G)$ ), is the 
total number of turns when both players play optimally and when Dominator (respectively Staller) starts the game.

Also, they investigated the relationship between domination number and game domination numbers of a graph. They proved that $\gamma(G) \leq \gamma_{g}(G) \leq 2 \gamma(G)-1$ for any graph $G$. In References [1,2], the authors showed that the two game domination numbers of a graph can differ by at most one. The domination game is being studied extensively since it was introduced. Kinnersley, West, and Zamani [2] posted the 3/5 conjecture: for any isolate-free graph $G, \gamma_{g}(G) \leq 3|V(G)| / 5$, and it has attracted several researches (See References [3-7]). In addition, there are researchers studying the domination game numbers on several classes of graphs such as paths, cycles, forests, disjoint union of graphs, split graphs, etc. (See References [4,8-11] for examples).

In 2015, a variation of the domination game called total domination game was introduced by Henning, Klavžar, and Rall [12]. In this version of the game, domination is replaced by total domination where a chosen vertex dominates its neighbors but not itself (See References [13-15]). The concept of total domination naturally gives rise to a variety of domination games such as Z-domination game, L-domination game, and LL-domination game (See Reference [16]). Many results in the domination game also hold for any of the above variations. For example, the difference between the number of moves in Dominator-start game and in Staller-start game is at most 1 for any of the above games.

In this paper, we are interested in studying the domination game such that the set of played vertices must be independent. In other words, a player can only play on an undominated vertex. Since an independent dominating set is a maximal independent set and vice versa, this independent version is the same as the competition-independence game which was introduced by Philips and Slater [17] in 2001.

The competition-independence game is played on a graph by two players, Diminisher and Sweller. They take turns in constructing maximal independent set $M$, where Diminisher tries to minimize $|M|$ and Sweller tries to maximize $|M|$. The competition-independence number, denoted by $I_{d}(G)$ (respectively $I_{S}(G)$ ), is the optimal size of the final independent set in the competition-independence game if Diminisher (respectively Sweller) starts the game. For the rest of the paper, we will use Dominator instead of Diminisher and Staller instead of Sweller.

Philips and Slater [18] provided the competition-independence numbers of a path. Consequently, the competition-independence numbers of a cycle of $n$ vertices established since the first move in $C_{n}$ produces $P_{n-3}$. In 2018, the competition-independence game in trees was studied by Goddard and Henning [19]. They provided the maximum and minimum values of the competition-independence game for trees of maximum degree 3.

In this paper, we check whether some well-known results in the domination game hold for the competition-independence game. We compare the competition-independence numbers to the game domination numbers. Also, we give some classes of graphs in which many parameters are equal. Finally, we establish a realization result of the competition-independence numbers.

\section{Relationship between the Competition-Independence Numbers and Other Parameters}

A fundamental tool for analyzing the original domination game is the Continuation Principle, which is proved in Reference [2]. We start this section by showing that the Continuation Principle for the domination game does not hold for the competition-independence game. Next, we compare the competition-independence numbers to the game domination numbers. Also, we provide a family of graphs such that many parameters are equal.

Theorem 1 ([2] (Continuation Principle)). Let $G$ be a (partially dominated) graph and let $A$ and $B$ be subsets of $V(G)$. Let $G_{A}$ and $G_{B}$ be partially dominated graphs in which the sets $A$ and $B$ have already been dominated, respectively. If $B \subseteq A$, then $\gamma_{g}\left(G_{A}\right) \leq \gamma_{g}\left(G_{B}\right)$ and $\gamma_{g}^{\prime}\left(G_{A}\right) \leq \gamma_{g}^{\prime}\left(G_{B}\right)$.

This result is very intuitive and natural. The more coverage the chosen vertices have, the fewer vertices are needed to be chosen to dominate the remaining undominated vertices. This result also holds for total domination game, Z-domination game, L-domination game, and LL-domination game [16]. 
However, it does not hold for the competition-independence game as the restriction of independence sometimes prevents players from making good moves. We present two simple counterexamples here.

Example 2. Consider the complete bipartite graph $K_{1,3}$ with vertex set $\left\{u, v_{1}, v_{2}, v_{3}\right\}$ and edge set $\left\{u v_{1}, u v_{2}, u v_{3}\right\}$. If $A=\left\{u, v_{1}\right\}$ and $B=\varnothing$, then $I_{d}\left(G_{A}\right)=2$ and $I_{d}\left(G_{B}\right)=1$.

Example 3. Let $G$ be the graph obtained from the complete bipartite graph $K_{3,3}$ with partite sets $V_{1}$ and $V_{2}$ by deleting a perfect matching. If $A=V_{1}$ and $B=\varnothing$, then $I_{S}\left(G_{A}\right)=3$ and $I_{S}\left(G_{B}\right)=2$.

Now, we compute the competition-independence numbers of complete multipartite graphs and complete bipartite graphs minus perfect matchings. These families of graphs will appear as examples throughout this paper.

Lemma 4. Let $n_{1}, n_{2}, \ldots, n_{m}$ be positive integers. Then, $I_{d}\left(K_{n_{1}, n_{2}, \ldots, n_{m}}\right)=\min \left\{n_{1}, n_{2}, \ldots, n_{m}\right\}$ and $I_{s}\left(K_{n_{1}, n_{2}, \ldots, n_{m}}\right)=\max \left\{n_{1}, n_{2}, \ldots, n_{m}\right\}$.

Proof. Let $V_{n_{1}}, V_{n_{2}}, \ldots, V_{n_{m}}$ be the partite sets of $K_{n_{1}, n_{2}, \ldots, n_{m}}$ with $\left|V_{n_{i}}\right|=n_{i}$ for $1 \leq i \leq m$. If a player starts the game by playing a vertex in $V_{n_{i}}$ for some $1 \leq i \leq m$, then both players must alternately choose vertices in the set $V_{n_{i}}$ so the game will end in $n_{i}$ moves. Hence, $I_{d}\left(K_{n_{1}, n_{2}, \ldots, n_{m}}\right)=\min \left\{n_{1}, n_{2}, \ldots, n_{m}\right\}$ and $I_{s}\left(K_{n_{1}, n_{2}, \ldots, n_{m}}\right)=\max \left\{n_{1}, n_{2}, \ldots, n_{m}\right\}$.

Lemma 5 . Let $G$ be the graph obtained from $K_{n, n}$ by deleting a perfect matching where $n \geq 2$. Then, $I_{d}(G)=n$ and $I_{s}(G)=2$.

Proof. Let $A=\left\{a_{1}, a_{2}, \ldots, a_{n}\right\}$ and $B=\left\{b_{1}, b_{2}, \ldots, b_{n}\right\}$ be the partite sets of the $K_{n, n}$, where $a_{i}$ and $b_{i}$ are not adjacent in $G$ for $i \in\{1,2, \ldots, n\}$.

We first show that $I_{d}(G)=n$. Without loss of generality, assume that Dominator starts the game by playing vertex $a_{1} \in A$. Then, each vertex in $B-\left\{b_{1}\right\}$ is dominated. Staller plays another vertex in $A$. Now all vertices in $B$ are dominated. Then, Dominator and Staller must alternately play all vertices in $A$. Consequently, $I_{d}(G)=n$.

We next show that $I_{s}(G)=2$. Without loss of generality, Staller starts the game by playing vertex $a_{1} \in A$. Then, Dominator finishes the game by playing $b_{1}$. Hence, $I_{s}(G)=2$.

The following four theorems show that the difference between the competition-independence numbers and the game domination numbers of a graph can be arbitrarily large.

Theorem 6. For a nonnegative integer $n$, there is a graph $G$ such that $I_{d}(G)-\gamma_{g}(G)=n$.

Proof. Consider $K_{n+3, t}$, where $t \geq n+3$. By Lemma $4, I_{d}\left(K_{n+3, t}\right)=n+3$. Note that $\gamma_{g}\left(K_{n+3, t}\right)=3$. Thus, $I_{d}\left(K_{n+3, t}\right)-\gamma_{g}\left(K_{n+3, t}\right)=n$.

Theorem 7. For a positive integer $n$, there is a graph $G$ such that $\gamma_{g}(G)-I_{d}(G)=n$.

Proof. Let $u$ be a vertex and $P_{9 n+1}=x_{1} y_{1} z_{1} x_{2} y_{2} z_{2} \ldots x_{3 n} y_{3 n} z_{3 n} x_{3 n+1}$ be a path. Define a graph $G$ by $V(G)=\{u\} \cup V\left(P_{9 n+1}\right)$ and $E(G)=\left\{u x_{j} \mid 1 \leq j \leq 3 n+1\right\} \cup E\left(P_{9 n+1}\right)$. See Figure 1 for example.

We first show that $I_{d}(G)=3 n+1$. To show that $I_{d}(G) \leq 3 n+1$, we present a strategy for Dominator. Dominator starts the game by playing the vertex $u$ first. Since $u x_{j} \in E(G)$ for all $1 \leq j \leq 3 n+1$, Staller and Dominator cannot play any vertex $x_{j}$ for all $1 \leq j \leq 3 n+1$. Thus, after the game ends, for each $j$, exactly one of $y_{j}$ or $z_{j}$ is played. Therefore, $3 n+1$ vertices are played and so $I_{d}(G) \leq 3 n+1$. To show that $I_{d}(G) \geq 3 n+1$, we present a strategy for Staller.

Case 1: Dominator starts the game by playing the vertex $u$.

Then, Staller plays $y_{1}$, and thus by similar arguments as above, after the game ends, $3 n+1$ vertices are played. Therefore, the number of moves in this case is at least $3 n+1$.

Case 2: Dominator starts the game by playing the vertex $y_{i}$ or $z_{i}$ for some $1 \leq i \leq 3 n$. 
Then, Staller plays the vertex $u$, and thus by similar arguments as above, after the game ends, $3 n+1$ vertices are played. Therefore, the number of moves in this case is at least $3 n+1$.

Case 3: Dominator starts the game by playing the vertex $x_{i}$ for some $1 \leq i \leq 3 n+1$.

Without loss of generality, we may assume that $1 \leq i \leq \frac{3 n+1}{2}$. Then, Staller plays his first move on vertex $y_{i+1}$. Consider the set $X:=\left\{x_{1}, x_{2}, x_{3}, \ldots, x_{3 n+1}\right\}-\left\{x_{i}, x_{i+1}\right\}$. Notice that each $x \in X$ is not dominated. In order to dominate $X$, an additional $3 n-1$ vertices are played. Thus, after the game ends, at least $3 n+1$ vertices are played.

From the above cases, $I_{d}(G) \geq 3 n+1$. Hence, $I_{d}(G)=3 n+1$.

We next show that $\gamma_{g}(G)=4 n+1$. To show that $\gamma_{g}(G) \geq 4 n+1$, we present a strategy for Staller.

Case 1: Dominator starts the game by playing the vertex $u$.

In each turn of Staller, he plays a vertex which dominates only one more undominated vertex. Since the vertex $x_{j}$ is dominated for all $1 \leq j \leq 3 n+1$, it follows that in each turn of Dominator, he can dominate at most two more undominted vertices. Consequently, in each round of the game played by Staller and then Dominator, there are at most three new dominated vertices. Since after the first move of Dominator at $u$ there are $9 n+2-(3 n+1)-1=6 n$ undominated vertices, it follows that the number of moves in this case is at least $\frac{2}{3} \cdot 6 n+1=4 n+1$.

Case 2: Dominator starts the game by playing a vertex $v$ where $v \neq u$.

Then, $v$ dominates at most four vertices including $v$ itself. Staller plays his first move on the vertex $u$, and after that, he plays to dominate one new undominated vertex at a time. Thus, for each $1 \leq j \leq 3 n+1, x_{j}$ is dominated. After that, for each round of the game, Dominator can dominate at most two more undominated vertices. Consequently, in each round of the game played by Dominator and then Staller, there are at most three new dominated vertices. After $u$ and $v$ are played, there are at least $(9 n+2)-4-(3 n+1)+1=6 n-2$ undominated vertices. Note that $(6 n-2)=(6 n-3)+1$. Therefore, the number of moves in this case is at least $\frac{2}{3} \cdot(6 n-3)+1+2=4 n+1$.

From the above cases, $\gamma_{g}(G) \geq 4 n+1$.

To show that $\gamma_{g}(G) \leq 4 n+1$, we present a strategy for Dominator. Dominator starts the game by playing the vertex $u$. For each turn of Dominator, he plays a vertex to dominate two new leftmost vertices if possible. Note that if Dominator cannot dominate two new vertices in his turn, that is he can dominate only one new vertex, then it means that Staller played a vertex to dominate two new vertices earlier. Therefore, on average, a move by Staller and a move by Dominator dominates at least three new vertices. Thus, $\gamma_{g}(G) \leq \frac{2}{3}(6 n)+1=4 n+1$. Consequently, $\gamma_{g}(G)=4 n+1$.

Therefore, $\gamma_{g}(G)-I_{d}(G)=(4 n+1)-(3 n+1)=n$, as required.

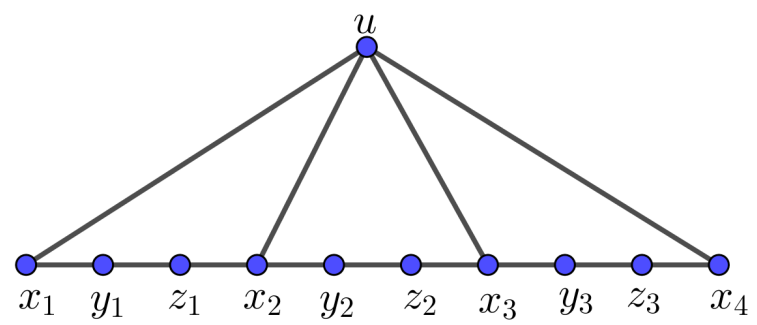

Figure 1. The graph $G$ in the proof of Theorem 7 , where $n=1$.

Theorem 8. For a nonnegative integer $n$, there is a graph $G$ such that $I_{S}(G)-\gamma_{g}^{\prime}(G)=n$.

Proof. Consider $K_{1, n+2}$. By Lemma $4, I_{s}\left(K_{1, n+2}\right)=n+2$. Note that $\gamma_{g}^{\prime}\left(K_{1, n+2}\right)=2$. Thus, $I_{s}\left(K_{1, n+2}\right)-$ $\gamma_{g}^{\prime}\left(K_{1, n+2}\right)=n$. 
Theorem 9. For a positive integer $n$, there is a graph $G$ such that $\gamma_{g}^{\prime}(G)-I_{s}(G)=n$.

Proof. Let $P_{9 n+1}=x_{1} y_{1} z_{1} x_{2} y_{2} z_{2} \ldots x_{3 n} y_{3 n} z_{3 n} x_{3 n+1}$ be a path and $K_{3 n+1}$ be a complete graph with $V\left(K_{3 n+1}\right)=\left\{u_{1}, u_{2}, \ldots, u_{3 n+1}\right\}$.

Define a graph $G$ by $V(G)=V\left(P_{9 n+1}\right) \cup V\left(K_{3 n+1}\right)$ and $E(G)=E\left(P_{9 n+1}\right) \cup E\left(K_{3 n+1}\right) \cup\left\{u_{j} x_{k} \mid j \neq\right.$ $k$ and $1 \leq j, k \leq 3 n+1\}$. See Figure 2 for example.

We first show that $\gamma_{g}^{\prime}(G)=4 n+2$. To show that $\gamma_{g}^{\prime}(G) \leq 4 n+2$, we present a strategy for Dominator.

Case 1: Staller starts the game by playing the vertex $u_{i}$ for some $1 \leq i \leq 3 n+1$.

Without loss of generality, we may assume that $1 \leq i \leq \frac{3 n+1}{2}$. Dominator responds by playing the vertex $y_{i}$. At this point, $y_{i}, z_{i}$ and all $u_{k}{ }^{\prime} \mathrm{s}(1 \leq k \leq 3 n+1)$ are dominated. After that, in each move of Dominator, he plays the leftmost vertex that can dominate two new vertices if possible. Note that, if Dominator cannot dominate two new vertices in his turn, that is he can dominate only one new vertex, then it means that Staller played a vertex to dominate two new vertices earlier. Therefore, excluding the first two moves, on average, a move by Staller and a move by Dominator dominate at least three new vertices. After Dominator plays $y_{i}$, there are $(12 n+2)-2(3 n+1)-2=6 n-2$ undominated vertices. Notice that $6 n-2=(6 n-3)+1=3(2 n-1)+1$. Hence, the number of moves in this case is at most $\frac{2}{3}(6 n-3)+1+2=4 n+1<4 n+2$.

Case 2: Staller starts the game by playing the vertex $y_{i}$ or $z_{i}$ for some $1 \leq i \leq 3 n$.

Without loss of generality, we may assume that Staller starts the game by playing the vertex $y_{i}$ for some $1 \leq i \leq 3 n$. Dominator responds by playing the vertex $u_{i}$ and so each vertex $x_{j}$ is dominated for $1 \leq j \leq 3 n+1$ and that each vertex $u_{k}$ is dominated for $1 \leq k \leq 3 n+1$. By similar arguments as in case 1 , after Dominator plays $u_{i}$, there are $(12 n+2)-2(3 n+1)-2=6 n-2$ undominated vertices and so the number of moves in this case is at most $4 n+2$.

Case 3: Staller starts the game by playing the vertex $x_{i}$ for some $1 \leq i \leq 3 n$.

Without loss of generality, we may assume that $1 \leq i \leq \frac{3 n+1}{2}$. Dominator responds by playing the vertex $u_{i}$ and so each vertex $x_{j}$ is dominated for $1 \leq j \leq 3 n+1$. By similar arguments as in case 1 , after Dominator plays $u_{i}$, there are at most $(12 n+2)-2(3 n+1)-1=6 n-1$ undominated vertices. Note that $6 n-1=(6 n-3)+2=3(2 n-1)+2$. Hence, the number of moves in this case is at most $\frac{2}{3}(6 n-3)+2+2=4 n+2$.

From the above cases, $\gamma_{g}^{\prime}(G) \leq 4 n+2$.

To show that $\gamma_{g}^{\prime}(G) \geq 4 n+2$, we present a strategy for Staller. Staller starts the game by playing the vertex $x_{1}$. Then, each vertex $u_{j}$ is dominated for $2 \leq j \leq 3 n+1$ and $y_{1}$ is also dominated.

Case 1: Dominator responds by playing the vertex $u_{1}$.

In each round of Staller's turn, he plays the leftmost vertex that can dominate one new vertex. Note that, in each Dominator's turn, he can dominate at most two new vertices. Therefore, excluding the first two moves, on average, a move by Staller and a move by Dominator dominate at most three new vertices. After Dominator plays $u_{1}$, there are $(12 n+2)-2(3 n+1)-1=6 n-1$ undominated vertices. Notice that $6 n-1=(6 n-3)+2=3(2 n-1)+2$. Therefore, the number of moves in this case is at least $\frac{2}{3}(6 n-3)+2+2=4 n+2$.

Case 2: Dominator responds by playing the vertex $w$ where $w \neq u_{1}$.

Then, Staller plays the vertex $u_{1}$ in his next turn, and after that, he will play the leftmost vertex that can dominate one new vertex. Note that, in each Dominator's turn, he can dominate at most two new vertices. Therefore, excluding the first three moves, on average, a move by Dominator and a move by Staller dominate at most three new vertices. After Staller plays $u_{1}$, there are at least 
$(12 n+2)-2(3 n+1)-2=6 n-2$ undominated vertices. Notice that $6 n-2=(6 n-3)+1=$ $3(2 n-1)+1$. Hence, the number of moves in this case is at least $\frac{2}{3}(6 n-3)+1+3=4 n+2$.

From the above cases, $\gamma_{g}^{\prime}(G) \geq 4 n+2$. Consequently, $\gamma_{g}^{\prime}(G)=4 n+2$.

We next show that $I_{s}(G)=3 n+2$. To show that $I_{s}(G) \leq 3 n+2$, we present a strategy for Dominator.

Case 1: Staller starts the game by playing a vertex $u_{i}$ for some $1 \leq i \leq 3 n+1$.

Dominator responds by playing the vertex $x_{i}$. After that, Staller and Dominator cannot play any vertex $u_{j}$ or $x_{j}$ for all $1 \leq j \leq 3 n+1$. For the remainder of the game, Staller and Dominator can only play either $y_{j}$ or $z_{j}$ for some $1 \leq j \leq 3 n$. After the game ends, for each $j$, exactly one of $y_{j}$ or $z_{j}$ is played for $1 \leq j \leq 3 n$. Therefore, $3 n+2$ vertices are played and so the number of moves in this case is at most $3 n+2$.

Case 2: Staller starts the game by playing a vertex $x_{i}$ for some $1 \leq i \leq 3 n+1$.

Dominator responses by playing the vertex $u_{i}$. By similar arguments as in case 1 , we have that $3 n+2$ vertices are played and so the number of moves in this case is at most $3 n+2$.

Case 3: Staller starts the game by playing either $y_{i}$ or $z_{i}$ for some $1 \leq i \leq 3 n$.

Dominator responds by playing the vertex $u_{i}$ if Staller played $y_{i}$; otherwise, he plays $u_{i+1}$. By similar arguments as in case 1 , we have that $(3 n-1)+2=3 n+1$ vertices are played. Therefore, the number of moves in this case is at most $3 n+1$.

From the above cases, $I_{s}(G) \leq 3 n+2$.

To show that $I_{S}(G) \geq 3 n+2$, we present a strategy for Staller. Staller starts the game by playing the vertex $x_{1}$.

Case 1: Dominator responds by playing the vertex $u_{1}$.

Then, the vertices $x_{j}$ and $u_{j}$ are dominated for $1 \leq j \leq 3 n+1$. Thus, after the game ends, for each $1 \leq j \leq 3 n$, exactly one of $y_{j}$ or $z_{j}$ is played. Therefore, $3 n+2$ vertices are played. Consequently, the number of moves in this case is at least $3 n+2$.

Case 2: Dominator responds by playing the vertex $y_{i}$ or $z_{i}$ for some $1 \leq i \leq 3 n$.

Without loss of generality, we may assume that Dominator responds by playing the vertex $y_{i}$ for some $1 \leq i \leq 3 n$. Then, Staller plays his next turn at the vertex $u_{1}$. At this point, the vertices $x_{j}$ and $u_{j}$ are dominated for $1 \leq j \leq 3 n+1$, and $y_{i}$ and $z_{i}$ are also dominated. Thus, after the game ends, for each $1 \leq k \leq 3 n$, exactly one of $y_{k}$ or $z_{k}$ is played. Hence, $3 n+2$ vertices are played. Consequently, the number of moves in this case is at least $3 n+2$.

Case 3: Dominator responds by playing the vertex $x_{i}$ for some $2 \leq i \leq 3 n+1$.

Then, each vertex $u_{j}$ is dominated for $1 \leq j \leq 3 n+1$. Note that, after Dominator plays $x_{i}$, there are at least $(12 n+2)-3 n-2-4=9 n-4$ undominated vertices. In each of Staller's turns, he plays to dominate at most two new undominated vertices. Notice that, in each turn of Dominator, he can dominate at most three new undominated vertices. Therefore, the number of moves is at least $\frac{2}{5}(9 n-4)+2=3 n+\frac{3}{5} n+\frac{2}{5}$. If $n \geq 2$, then the number of moves is at least $3 n+2$. For $n=1$, it can be checked that the number of moves is at least 5 .

From the above cases, $I_{s}(G) \geq 3 n+2$. Consequently, $I_{s}(G)=3 n+2$.

Therefore, $\gamma_{g}^{\prime}(G)-I_{s}(G)=4 n+2-(3 n+2)=n$.

By the definitions, $i(G) \leq I_{d}(G) \leq \alpha(G)$ and $i(G) \leq I_{s}(G) \leq \alpha(G)$. We show that the difference between the competition-independence numbers and independent domination numbers can be arbitrarily large.

Theorem 10. For a nonnegative integer $n$, there is a graph $G$ such that $I_{d}(G)-i(G)=n$. 


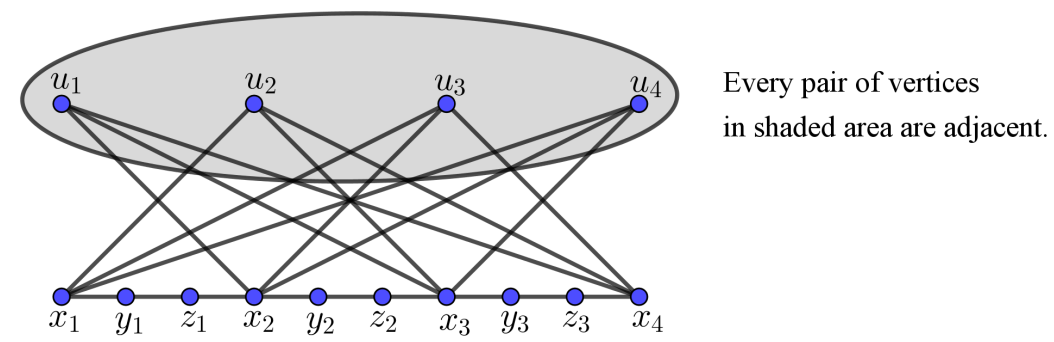

Figure 2. The graph $G$ in the proof of Theorem 9, where $n=1$.

Proof. Let $G$ be the graph obtained from $K_{n+2, n+2}$ by deleting a perfect matching. By Lemma 5, we have $I_{d}(G)=n+2$. Note that $i(G)=2$. Thus $I_{d}(G)-i(G)=n$.

Theorem 11. For a nonnegative integer $n$, there is a graph $G$ such that $\alpha(G)-I_{d}(G)=n$.

Proof. Consider $K_{1, n+1}$. Note that $\alpha\left(K_{1, n+1}\right)=n+1$ and $I_{d}\left(K_{1, n+1}\right)=1$. Thus $\alpha\left(K_{1, n+1}\right)-I_{d}\left(K_{1, n+1}\right)=n$.

Theorem 12. For a nonnegative integer $n$, there is a graph $G$ such that $I_{s}(G)-i(G)=n$.

Proof. Consider $K_{1, n+1}$. Note that $I_{s}\left(K_{1, n+1}\right)=n+1$ and $i\left(K_{1, n+1}\right)=1$. Thus $I_{s}\left(K_{1, n+1}\right)-i\left(K_{1, n+1}\right)=n$.

Theorem 13. For a nonnegative integer $n$, there is a graph $G$ such that $\alpha(G)-I_{s}(G)=n$.

Proof. Let $G$ be the graph obtained from $K_{n+2, n+2}$ by deleting a perfect matching. Note that $\alpha(G)=n+2$ and $I_{s}(G)=2$. Thus, $\alpha(G)-I_{S}(G)=n$.

Next, we show a family of graphs such that many parameters are equal.

The corona product of graphs $G$ and $H$, denoted by $G \circ H$, is a graph obtained by taking one copy of $G$ and $|V(G)|$ copies of $H$ and by joining each vertex of the $i$ th copy of $H$ to the $i$ th vertices of $G$, where $1 \leq i \leq|V(G)|$.

Theorem 14. There exists a graph $G$ such that $I_{d}(G)=I_{s}(G)=\gamma_{g}(G)=\gamma_{g}^{\prime}(G)=\alpha(G)=i(G)$.

Proof. Let $G$ be a graph $K_{n} \circ K_{1}$. Note that $I_{d}(G)=I_{s}(G)=\gamma_{g}(G)=\gamma_{g}^{\prime}(G)=\alpha(G)=i(G)=n$.

\section{Realization of the Competition-Independence Numbers}

For a pair $(a, b)$ of positive integers, we say $G$ realizes $(a, b)$ if $I_{d}(G)=a$ and $I_{S}(G)=b$. In this section, we show that any pair $(a, b)$ of positive integers can be realized.

Theorem 15. For positive integers $a$ and $b$, there is a connected graph $G$ such that $I_{d}(G)=a$ and $I_{s}(G)=b$.

Proof. Let $a$ and $b$ be positive integers.

Case 1: $a \leq b$.

Let $G$ be the complete bipartite graph $K_{a, b}$. By Lemma 4, we have $I_{d}(G)=\min \{a, b\}=a$ and $I_{S}(G)=\max \{a, b\}=b$.

Case 2: $a>b$.

Let $H$ be the complete $b$-partite graph such that the size of each partite set is $a$ i.e., $H=K_{a, a, \ldots, a}$. Suppose that $V_{1}, V_{2}, \ldots, V_{b}$ are the partite sets of $H$ and that $V_{i}=\left\{v_{i, 1}, v_{i, 2}, \ldots, v_{i, a}\right\}$ for $1 \leq i \leq b$. Let $G$ be the graph $H-\left\{v_{i, j} v_{k, j} \mid 1 \leq i<k \leq b\right.$ and $\left.1 \leq j \leq a\right\}$.

Let $T:=\left\{v_{k, 1} \mid 2 \leq k \leq b\right\}$. We first show that $I_{d}(G)=a$.

Without loss of generality, we may assume that Dominator starts the game by playing vertex $v_{1,1}$. Then, the set $\left(V_{1}-\left\{v_{1,1}\right\}\right) \cup T$ is the set of all undominated vertices. Since $a>b$, Staller responds by 
playing a vertex in $V_{1}-\left\{v_{1,1}\right\}$, and then, both players must alternately choose undominated vertices in the set $V_{1}$. Therefore, the game will end in $a$ moves. Hence, $I_{d}(G)=a$.

We next show that $I_{S}(G)=b$.

Without loss of generality, we may assume that Staller starts the game by playing vertex $v_{1,1}$. Then, the set $\left(V_{1}-\left\{v_{1,1}\right\}\right) \cup T$ is the set of all undominated vertices. Since $a>b$, Dominator responds by playing a vertex in the set $T$, and then, both players must alternately choose undominated vertices in the set $T$. Therefore, the game will end in $b$ moves. Hence, $I_{S}(G)=b$.

In particular, Theorem 15 shows that the difference between the two competition-independence numbers of a graph can be arbitrarily large. This is very different from the domination game and the other variations where the difference is at most one $[1,2,16]$.

\section{Conclusions}

In this paper, we studied the domination game such that the set of played vertices is independent. This game is known as the competition-independence game. We showed that the Continuation Principle for the domination game does not hold for the competition-independence game. We proved that the difference between the competition-independence numbers and the game domination numbers of a graph can be arbitrarily large. Also, we gave a family of graphs such that many parameters are equal. Furthermore, we showed that any pair of positive integers can be realized as the competition-independence numbers of some graph.

Author Contributions: Investigation, W.R.; writing-original draft preparation, W.R.; writing-review and editing, C.W.; supervision, C.W. All authors have read and agreed to the published version of the manuscript.

Funding: This research was funded by The Thailand Research Fund (grant number MRG6180301).

Acknowledgments: The authors would like to thank the reviewers for their suggestions and comments. The second author was supported by The Thailand Research Fund (grant number MRG6180301). The author takes this oppotunity to thank The Thailand Research Fund for the support.

Conflicts of Interest: The authors declare no conflict of interest.

\section{References}

1. Brešar, B.; Klavžar, S.; Rall, D.F. Domination game and an imagination strategy. SIAM J. Discret. Math. 2010, 24, 979-991. [CrossRef]

2. Kinnersley, W.B.; West, D.B.; Zamani, R. Extremal problems for game domination number. SIAM J. Discret. Math. 2013, 27, 2090-2107. [CrossRef]

3. Brešar, B.; Klavžar, S.; Košmrlj G.; Rall, D.F. Domination game: Extremal families of graphs for the 3/5-conjectures. Discret. Appl. Math. 2013, 161, 1308-1316. [CrossRef]

4. Bujtas, C. Domination game on forests. Discret. Math. 2015, 338, 2220-2228. [CrossRef]

5. Bujtas, C. On the game domination number of graphs with given minimum degree. Electron. J. Combin. 2015, 22, 3.29. [CrossRef]

6. Henning, M.A.; Kinnersley, W.B. Domination game: A proof of the 3/5-conjecture for graphs with minimum degree at least two. SIAM J. Discret. Math. 2016, 30, 20-35. [CrossRef]

7. Henning, M.A.; Löwenstein, C. Domination game: Extremal families for the 3/5-conjecture for forests. Discuss. Math. Graph Theory 2017, 37, 369-381. [CrossRef]

8. Dorbec, P.; Košmrlj, G.; Renault, G. The domination game played on unions of graphs. Discret. Math. 2015, 338, 71-79. [CrossRef]

9. James, T.; Klavžar, S.; Vijayakumar, A. The domination game on split graphs, Bull. Aust. Math. Soc. 2019, 99, $327-337$. [CrossRef]

10. Ruksasakchai, W.; Onphaeng, K.; Worawannotai, C. Game domination numbers of a disjoint union of paths and cycles. Quaest. Math. 2019, 42, 1357-1372. [CrossRef]

11. Zamani, R. Hamiltonian cycles through specified edges in bipartite graphs, domination game, and the game of revolutionaries and spies. Ph.D. Thesis, University of Illinois at Urbana-Champaign, IL, USA. 
12. Henning, M.A.; Klavžar, S.; Rall, D.F. Total version of the domination game. Graphs Combin. 2015, 31, $1453-1462$. [CrossRef]

13. Bujtas, C. On the game total domination number. Graphs Comb. 2018, 34, 415-425. [CrossRef]

14. Dorbec, P.; Henning, M.A. Game total domination for cycles and paths. Discret. Appl. Math. 2016, 208, 7-18. [CrossRef]

15. Henning, M.A.; Klavžar, S.; Rall, D.F. The 4/5 upper bound on the game total domination number. Combinatorica 2017, 37, 223-251. [CrossRef]

16. Brešar, B.; Bujtas, C.; Gologranc, T.; Klavžar, S.; Košmrlj, G.; Marc, T.; Patkos, B.; Tuza, Z.; Vizer, M. The variety of domination games. Aequ. Math. 2019, 93, 1085-1109. [CrossRef]

17. Phillips, J.B.; Slater, P.J. An introduction to graph competition independence and enclaveless parameters. Graph. Theory Notes N.Y. 2001, 41, 37-41.

18. Phillips, J.B.; Slater, P.J. Graph competition independence and enclaveless parameters. Congr. Numer. 2002, 154, 79-100.

19. Goddard, W.; Henning, M.A. The competition-independence game in trees. J. Combin. Math. Combin. Comput. 2018, 104, 161-170.

(C) 2020 by the authors. Licensee MDPI, Basel, Switzerland. This article is an open access article distributed under the terms and conditions of the Creative Commons Attribution (CC BY) license (http://creativecommons.org/licenses/by/4.0/). 